\title{
Solving an Integral Equation by Using Fixed Point Approach in Fuzzy Bipolar Metric Spaces
}

\author{
Gunaseelan Mani $\mathbb{D}^{1},{ }^{1}$ Arul Joseph Gnanaprakasam $\mathbb{D}^{\mathbb{D}},{ }^{2}$ Absar U1 Haq, ${ }^{3}$ Fahd Jarad $\mathbb{D},{ }^{4,5}$ \\ and Imran Abbas Baloch ${ }^{6,7}$ \\ ${ }^{1}$ Department of Mathematics, Saveetha School of Engineering, Saveetha Institute of Medical and Technical Sciences, \\ Saveetha University, Chennai 602 105, India \\ ${ }^{2}$ Department of Mathematics, College of Engineering and Technology, Faculty of Engineering and Technology, SRM Institute of \\ Science and Technology, SRM Nagar, Kattankulathur 603203, Kanchipuram, Chennai, Tamil Nadu, India \\ ${ }^{3}$ Department of Natural Sciences and Humanities, University of Engineering and Technology (Narowal Campus), \\ Lahore 54000, Pakistan \\ ${ }^{4}$ Department of Mathematics, Çankaya University, Etimesgut, 06790 Ankara, Turkey \\ ${ }^{5}$ Department of Medical Research, China Medical University, Taichung 40402, Taiwan \\ ${ }^{6}$ Abdus Salam School of Mathematical Sciences, GC University, Lahore, Pakistan \\ ${ }^{7}$ Higher Education Department, Government College for Boys Gulberg, Punjab, Pakistan
}

Correspondence should be addressed to Fahd Jarad; fahd@cankaya.edu.tr and Imran Abbas Baloch; iabbasbaloch@sms.edu.pk

Received 14 September 2021; Revised 26 October 2021; Accepted 22 November 2021; Published 3 December 2021

Academic Editor: Anita Tomar

Copyright (c) 2021 Gunaseelan Mani et al. This is an open access article distributed under the Creative Commons Attribution License, which permits unrestricted use, distribution, and reproduction in any medium, provided the original work is properly cited.

The purpose of this manuscript is to obtain some fixed point results under mild contractive conditions in fuzzy bipolar metric spaces. Our results generalize and extend many of the previous findings in the same approach. Moreover, two examples to support our theorems are obtained. Finally, to examine and strengthen the theoretical results, the existence and uniqueness of the solution to a nonlinear integral equation was studied as a kind of applications.

\section{Introduction}

The notion of the continuous triangular norm was introduced in 1960 by Schweizer and Sklar in their paper [1]. The concept of fuzzy set theory was initiated by Zadeh [2] in 1965. Some references to a fuzzy logic-based education system can be found in [3-6]. The other direction of the fuzzy set is the fuzzy metric theory. The idea of fuzzy metric space (FM-space) was presented by Kramosil and Michalek [7]. With the help of continuous $t$-norm property, they obtained some pivotal fixed point results under the mild contractive conditions in the mentioned space. Many authors worked in this direction; they either modified the definition of FM-spaces [8] or extended the well-known fixed point theorem of Banach to fuzzy metric spaces [9]. Moreover, Gregori and Sapena $[5,10]$ obtained some contractive-type fixed point theorems in FM-spaces. Recently, in 2020, Li et al. [11] showed some strongly coupled fixed point theorems by using cyclic contractivetype mappings in complete FM-spaces. In 2019, Beloul and Tomar [12] proved integral-type common fixed point theorems in modified intuitionistic fuzzy metric spaces. Prasad et al. [13] presented coincidence theorems via contractive mappings in ordered non-Archimedean fuzzy metric spaces. Again Prasad [14] analyzed coincidence points of relational $\psi$-contractions in 2021. The bipolar metric space has been studied by many authors, and important results have been obtained [15-18].

Recently, FM-space was extended and generalized to fuzzy bipolar metric space (FBM-space) by Mutlu and Gurdal [19]. They gave new concepts for measurement of the distance between the elements of two different sets. Bartwal 
et al. [20] introduced the notion of fuzzy bipolar metric space and obtained some fixed point results under mild conditions.

A continuation of this approach, in this manuscript, we shall obtain some fixed point theorems via contractive-type mappings in FBM-spaces. Our results generalize, unify, and extend the results of Bartwal et al. [20] and many other papers in this direction. Also, two examples are given to support our theorems. Ultimately, the existence and uniqueness solution to an integral equation in the sense of Lebesgue measurable functions are obtained as an application.

\section{Basic Facts}

This part is devoted to present some basic definitions, lemmas, and propositions of FBM-spaces as follows.

Definition 1. (see [8]). Let $\Pi$ be a nonvoid set. A 3-triple $(\Omega$, $\Gamma$,*) is called an FM-space if $\Gamma$ is a fuzzy set on $\Omega^{2} \times(0, \infty)$ and $*$ is a continuous $\eta$-norm justifying the hypotheses below:

(1) $\Gamma(\mu, \sigma, \eta)>0$

(2) $\Gamma(\mu, \sigma, \eta)=1$ iff $\mu=\sigma$

(3) $\Gamma(\mu, \sigma, \eta)=\Gamma(\sigma, \mu, \eta)$

(4) $\Gamma(\mu, \vartheta, \eta+\zeta) \geq \Gamma(\mu, \sigma, \eta) * \Gamma(\sigma, \vartheta, \zeta)$

(5) $\Gamma(\mu, \sigma,):.(0, \infty) \longrightarrow(0,1]$ is continuous

for all $\mu, \sigma, \vartheta \in \Omega$ and $\eta, \zeta>0$.

Lemma 2. (see [21]). Let $(\Pi, \Gamma$,*) be an FM-space. If for all $\mu, \sigma \in \Pi$ and $\eta>0$.

$$
\Gamma(\mu, \sigma, k \eta) \geq \Gamma(\mu, \sigma, \eta)
$$

where $k \in(0,1)$, then $\mu=\sigma$.

Definition 3. (see [20]). Let $\Pi$ and $\Omega$ be two nonvoid sets. A 4-tuple $\left(\Pi, \Omega, \Gamma_{\mathfrak{b}}, *\right)$ is said to be an FBM-space, where $*$ is continuous $\eta$-norm and $\Gamma_{\mathfrak{b}}$ is a fuzzy set on $\Pi \times \Omega \times(0, \infty)$, fulfilling the subsequent assumptions:

(1) $\Gamma_{\mathfrak{b}}(\mu, \sigma, \eta)>0$ for all $(\mu, \sigma) \in \Pi \times \Omega$

(2) $\Gamma_{\mathfrak{b}}(\mu, \sigma, \eta)=1$ iff $\mu=\sigma$ for $\mu \in \Pi$ and $\sigma \in \Omega$

(3) $\Gamma_{\mathfrak{b}}(\mu, \sigma, \eta)=\Gamma_{\mathfrak{b}}(\sigma, \mu, \eta)$ for all $\mu, \sigma \in \Pi \cap \Omega$

(4) $\Gamma_{\mathfrak{b}}\left(\mu_{1}, \sigma_{2}, \eta+\zeta+r\right) \geq \Gamma_{\mathfrak{b}}\left(\mu_{1}, \sigma_{1}, \eta\right) * \Gamma_{\mathfrak{b}}\left(\mu_{2}, \sigma_{1}, \zeta\right) *$ $\Gamma_{\mathfrak{b}}\left(\mu_{2}, \sigma_{2}, r\right)$ for all $\mu_{1}, \mu_{2} \in \Pi$ and $\sigma_{1}, \sigma_{2} \in \Omega$

(5) $\Gamma_{\mathfrak{b}}(\mu, \sigma,):.[0, \infty) \longrightarrow[0,1]$ is left continuous

(6) $\Gamma_{\mathfrak{b}}(\mu, \sigma,$.$) is nondecreasing for all \mu \in \Pi$ and $\sigma \in \Omega$, for all $\eta, \zeta, r>0$

Remark 4. (see [20]). In an FBM-space $\left(\Pi, \Omega, \Gamma_{\mathfrak{b}}, *\right)$, if $\Pi$ $=\Omega$, then $\left(\Pi, \Gamma_{\mathfrak{b}}, *\right)$ is an FM-space.
Lemma 5. (see [20]). Let $\left(\Pi, \Omega, \Gamma_{\mathfrak{b}}, *\right)$ be an FBM-space so that

$$
\Gamma_{\mathfrak{b}}(\mu, \sigma, k \eta) \geq \Gamma_{\mathfrak{b}}(\mu, \sigma, \eta)
$$

for $\mu \in \Pi, \sigma \in \Omega$ and $k \in(0,1)$. Then, $\mu=\sigma$.

Definition 6. (see [20]). Let $\left(\Pi, \Omega, \Gamma_{\mathfrak{b}}, *\right)$ be an FBM-space. A point $\sigma \in \Pi \cup \Omega$ is called a left point if $\sigma \in \Pi$, a right point if $\sigma \in \Omega$, and a central point if it is both a left and a right point. Similarly, a sequence $\left\{\sigma_{\alpha}\right\}$ on the set $\Pi$ is called a left sequence, and a sequence $\left\{\sigma_{\alpha}\right\}$ on $\Omega$ is called a right sequence. In an FBM-space, a left or a right sequence is called simply a sequence. A sequence $\left\{\sigma_{\alpha}\right\}$ is said to be convergent to a point $\sigma$, iff $\left\{\sigma_{\alpha}\right\}$ is a left sequence, $\sigma$ is a right point, and $\lim _{\alpha \longrightarrow \infty} \Gamma_{\mathfrak{b}}\left(\sigma_{\alpha}, \sigma, \eta\right)=1$. A bisequence $\left(\left\{\sigma_{\alpha}\right\},\{\right.$ $\left.\left.\mu_{\alpha}\right\}\right)$ on $\left(\Pi, \Omega, \Gamma_{\mathfrak{b}}, *\right)$ is a sequence on the set $\Pi \times \Omega$. If the sequence $\left\{\sigma_{\alpha}\right\}$ and $\left\{\mu_{\alpha}\right\}$ are convergent, then the bisequence $\left(\left\{\sigma_{\alpha}\right\},\left\{\mu_{\alpha}\right\}\right)$ is said to be convergent, and if $\left\{\sigma_{\alpha}\right\}$ and $\left\{\mu_{\alpha}\right\}$ converge to a common point, then $\left(\left\{\sigma_{\alpha}\right\},\left\{\mu_{\alpha}\right\}\right)$ is called biconvergent. A bisequence $\left(\left\{\sigma_{\alpha}\right\},\left\{\mu_{\alpha}\right\}\right)$ is a Cauchy bisequence, if $\lim _{\alpha, \beta \rightarrow \infty} \Gamma_{\mathfrak{b}}\left(\sigma_{\alpha}, \mu_{\beta}, \eta\right)=1$. An FBMspace is called complete, if every Cauchy bisequence is convergent, hence biconvergent.

Lemma 7. (see [20]). In an FBM-space, every convergent Cauchy bisequence is biconvergent.

Lemma 8. (see [20]). Let $\left(\Pi, \Omega, \Gamma_{\mathfrak{b}}, *\right)$ be an FBM-space, and if $\mu \in \Pi \cap \Omega$ is a limit of a sequence, then it is a unique limit of the sequence.

Definition 9. A point $\mu \in \Pi \cap \Omega$ is said to be common fixed point for the mappings $(\Lambda, \Theta)$ on $\mu \in \Pi \cap \Omega$ such that $\mu=$ $\Lambda \mu=\Theta \mu$.

\section{Main Results}

Now, we present the first main theorem.

Theorem 10. Let $\left(\Pi, \Omega, \Gamma_{\mathfrak{b}}\right.$,*) be a complete FBM-space such that

$$
\lim _{\eta \longrightarrow \infty} \Gamma_{\mathfrak{b}}(\mu, \sigma, \eta)=1 \quad \text { for all } \mu \in \Pi, \sigma \in \Omega \text {. }
$$

Let $\Lambda, \Theta: \Pi \cup \Omega \longrightarrow \Pi \cup \Omega$ be two mappings satisfying

(1) $\Lambda(\Pi) \subseteq \Pi, \Theta(\Pi) \subseteq \Pi$, and $\Lambda(\Omega) \subseteq \Omega, \Theta(\Omega) \subseteq \Omega$

(2) $\Gamma_{\mathfrak{b}}(\Lambda(\mu), \Theta(\sigma), k \eta) \geq \Gamma_{\mathfrak{b}}(\mu, \sigma, \eta)$ for all $\mu \in \Pi, \sigma \in \Omega$ and $\eta>0$, where $0<k<1$

Then, $\Lambda$ and $\Theta$ have a unique common fixed point.

Proof. Fix $\mu_{0} \in \Pi$ and $\sigma_{0} \in \Omega$ and assume that $\Lambda\left(\mu_{2 \alpha}\right)=\mu_{2 \alpha+1}$, $\Theta\left(\mu_{2 \alpha+1}\right)=\mu_{2 \alpha+2}, \Lambda\left(\sigma_{2 \alpha}\right)=\sigma_{2 \alpha+1}$, and $\Theta\left(\sigma_{2 \alpha+1}\right)=\sigma_{2 \alpha+2}$ for all $\alpha \in \mathbb{N} \cup\{0\}$. Then, we get $\left(\mu_{\alpha}, \sigma_{\alpha}\right)$ as a bisequence on the 
FBM-space $\left(\Pi, \Omega, \Gamma_{\mathfrak{b}}, *\right)$. Now, we have

$$
\Gamma_{\mathfrak{b}}\left(\mu_{1}, \sigma_{1}, \eta\right)=\Gamma_{\mathfrak{b}}\left(\Lambda\left(\mu_{0}\right), \Theta\left(\sigma_{0}\right), \eta\right) \geq \Gamma_{\mathfrak{b}}\left(\mu_{0}, \sigma_{0}, \frac{\eta}{k}\right)
$$

$\forall \eta>0$ and $\alpha \in \mathbb{N}$. By induction, we obtain

$$
\begin{gathered}
\Gamma_{\mathfrak{b}}\left(\mu_{2 \alpha+1}, \sigma_{2 \alpha+1}, \eta\right)=\Gamma_{\mathfrak{b}}\left(\Lambda\left(\mu_{2 \alpha}\right), \Theta\left(\sigma_{2 \alpha}\right), \eta\right) \geq \Gamma_{\mathfrak{b}}\left(\mu_{0}, \sigma_{0}, \frac{\eta}{k^{2 \alpha+1}}\right) \\
\Gamma_{\mathfrak{b}}\left(\mu_{2 \alpha+1}, \sigma_{2 \alpha+2}, \eta\right)=\Gamma_{\mathfrak{b}}\left(\Lambda\left(\mu_{2 \alpha}\right), \Theta\left(\sigma_{2 \alpha+1}\right), \eta\right) \geq \Gamma_{\mathfrak{b}}\left(\mu_{0}, \sigma_{1}, \frac{\eta}{k^{2 \alpha+1}}\right)
\end{gathered}
$$

for all $\eta>0$ and $\alpha \in \mathbb{N}$.

Letting $\alpha<\beta$, for $\alpha, \beta \in \mathbb{N}$. Then, from the definition of the FBM-space, we get

$$
\begin{aligned}
& \Gamma_{\mathfrak{b}}\left(\mu_{\alpha}, \sigma_{\beta}, \eta\right) \geq \Gamma_{\mathfrak{b}}\left(\mu_{\alpha}, \sigma_{\alpha}, \frac{\eta}{3}\right) * \Gamma_{\mathfrak{b}}\left(\mu_{\alpha+1}, \sigma_{\alpha}, \frac{\eta}{3}\right) \\
& * \Gamma_{\mathfrak{b}}\left(\mu_{\alpha+1}, \sigma_{\beta}, \frac{\eta}{3}\right) \\
& \vdots \\
& \geq \Gamma_{\mathfrak{b}}\left(\mu_{\alpha}, \sigma_{\alpha}, \frac{\eta}{3}\right) * \Gamma_{\mathfrak{b}}\left(\mu_{\alpha+1}, \sigma_{\alpha}, \frac{\eta}{3}\right) * \cdots \\
& \quad * \Gamma_{\mathfrak{b}}\left(\mu_{\beta-1}, \sigma_{\beta-1}, \frac{\eta}{3^{\beta-1}}\right) * \Gamma_{\mathfrak{b}}\left(\mu_{\beta}, \sigma_{\beta-1}, \frac{\eta}{3^{\beta-1}}\right) \\
& \quad * \Gamma_{\mathfrak{b}}\left(\mu_{\beta}, \sigma_{\beta}, \frac{\eta}{3^{\beta-1}}\right) .
\end{aligned}
$$

Therefore,

$$
\begin{aligned}
\Gamma_{\mathfrak{b}}\left(\mu_{\alpha}, \sigma_{\beta}, \eta\right) \geq & \Gamma_{\mathfrak{b}}\left(\mu_{0}, \sigma_{0}, \frac{\eta}{3 k^{\alpha}}\right) * \Gamma_{\mathfrak{b}}\left(\mu_{0}, \sigma_{1}, \frac{\eta}{3 k^{\alpha+1}}\right) * \cdots \\
& \cdots * \Gamma_{\mathfrak{b}}\left(\mu_{0}, \sigma_{0}, \frac{\eta}{3^{\beta-1} k^{\beta+1}}\right) .
\end{aligned}
$$

From (3), as $\alpha, \beta \longrightarrow \infty$, we get

$$
\Gamma_{\mathfrak{b}}\left(\mu_{\alpha}, \sigma_{\beta}, \eta\right) \geq 1 \quad \forall \eta>0
$$

Thus, bisequence $\left(\left\{\mu_{\alpha}\right\},\left\{\sigma_{\alpha}\right\}\right)$ is a Cauchy bisequence. Since $\left(\Pi, \Omega, \Gamma_{\mathfrak{b}}, *\right)$ is a complete FBM-space. By Lemma 7 , bisequence $\left(\left\{\mu_{\alpha}\right\},\left\{\sigma_{\alpha}\right\}\right)$ is a biconvergent sequence. Therefore, $\left\{\mu_{\alpha}\right\} \longrightarrow u$ and $\left\{\sigma_{\alpha}\right\} \longrightarrow u$, where $u \in \Pi \cap \Omega$. By Lemma 8 , both sequences $\left\{\mu_{\alpha}\right\}$ and $\left\{\sigma_{\alpha}\right\}$ have a unique limit. From the triangular property of fuzzy bipolar metric spaces, we have

$$
\begin{aligned}
& \Gamma_{\mathfrak{b}}(\Lambda(u), u, \eta) \geq \Gamma_{\mathfrak{b}}\left(\Lambda(u), \sigma_{\alpha+1}, \frac{\eta}{3}\right) * \Gamma_{\mathfrak{b}}\left(\mu_{\alpha+1}, \sigma_{\alpha+1}, \frac{\eta}{3}\right) \\
& * \Gamma_{\mathfrak{b}}\left(\mu_{\alpha+1}, u, \frac{\eta}{3}\right)=\Gamma_{\mathfrak{b}}\left(\Lambda(u), \Theta\left(\sigma_{\alpha}\right), \frac{\eta}{3}\right) \\
& * \Gamma_{\mathfrak{b}}\left(\mu_{\alpha+1}, \sigma_{\alpha+1}, \frac{\eta}{3}\right) * \Gamma_{\mathfrak{b}}\left(\mu_{\alpha+1}, u, \frac{\eta}{3}\right) \geq \Gamma_{\mathfrak{b}}\left(u, \sigma_{\alpha}, \frac{\eta}{3}\right) \\
& \quad * \Gamma_{\mathfrak{b}}\left(\mu_{\alpha+1}, \sigma_{\alpha+1}, \frac{\eta}{3}\right) * \Gamma_{\mathfrak{b}}\left(\mu_{\alpha+1}, u, \frac{\eta}{3}\right),
\end{aligned}
$$

for all $\alpha \in \mathbb{N}$ and $\eta>0$ and as $\alpha \longrightarrow \infty$,

$$
\Gamma_{\mathfrak{b}}(\Lambda(u), u, \eta) \longrightarrow 1 * 1 * 1=1
$$

From Definition 3 condition $(2), \Lambda(u)=u$. Again,

$$
\Gamma_{\mathfrak{b}}(u, \Theta(u), \eta)=\Gamma_{\mathfrak{b}}(\Lambda(u), \Theta(u), \eta) \geq \Gamma_{\mathfrak{b}}\left(u, u, \frac{\eta}{k}\right)=1
$$

Therefore, $\Theta(u)=u$. Hence, $u$ is a common fixed point of $\Lambda$ and $\Theta$.

Let $v \in \Pi \cap \Omega$ be another fixed point of $\Lambda$ and $\Theta$. Then,

$$
\Gamma_{\mathfrak{b}}(u, v, \eta)=\Gamma_{\mathfrak{b}}(\Lambda(u), \Theta(v), \eta) \geq \Gamma_{\mathfrak{b}}\left(u, v, \frac{\eta}{k}\right)
$$

for $0<k<1$ and $\forall \eta>0$. By Lemma 5 , we have $u=v$.

The following example supports the above theorem.

Example 11. Let $\Pi=[0,2]$ and $\Omega=\{0\} \cup \mathbb{N}-\{1,2\}$. Define $\Gamma_{\mathfrak{b}}=\eta /(\eta+|\mu-\sigma|)$ for all $\eta>0, \mu \in \Pi$, and $\sigma \in \Omega$. Clearly, $\left(\Pi, \Omega, \Gamma_{\mathfrak{b}}, *\right)$ is a complete FBM-space, where $*$ is a continuous $\eta$-norm defined as $\mathfrak{p} * \mathfrak{q}=\mathfrak{p} \mathfrak{q}$.

Let $\Lambda, \Theta: \Pi \cup \Omega \longrightarrow \Pi \cup \Omega$ be mappings defined by

$$
\begin{aligned}
& \Lambda(\mu)=\left(\begin{array}{ll}
2-\mu, & \text { if } \mu \in[0,2], \\
2, & \text { if } \mu \in \mathbb{N}-\{1,2\},
\end{array}\right. \\
& \Theta(\mu)=\left(\begin{array}{ll}
\mu, & \text { if } \mu \in[0,2], \\
2, & \text { if } \mu \in \mathbb{N}-\{1,2\},
\end{array}\right.
\end{aligned}
$$

for all $\mu \in \Pi \cup \Omega$. Now, suppose that $k=1 / 2$, then for all $\eta>0$, we discuss the following cases:

Case 1. If $\mu \in[0,2]$ and $\sigma \in \mathbb{N}-\{1,2\}$, then

$$
\begin{aligned}
\Gamma_{\mathfrak{b}}(\Lambda(\mu), \Theta(\sigma), k \eta) & =\Gamma_{\mathfrak{b}}(2-\mu, 2, k \eta)=\frac{k \eta}{k \eta+|2-\mu-2|} \\
& \geq \frac{\eta}{\eta+|\mu-\sigma|}=\Gamma_{\mathfrak{b}}(\mu, \sigma, \eta) .
\end{aligned}
$$


Case 2. If $\mu \in \mathbb{N}-\{1,2\}$ and $\sigma \in[0,2]$, then

$$
\begin{aligned}
\Gamma_{\mathfrak{b}}(\Lambda(\mu), \Theta(\sigma), k \eta) & =\Gamma_{\mathfrak{b}}(2, \sigma, k \eta)=\frac{k \eta}{k \eta+|2-\sigma|} \\
& \geq \frac{\eta}{\eta+|\mu-\sigma|}=\Gamma_{\mathfrak{b}}(\mu, \sigma, \eta)
\end{aligned}
$$

Therefore, the conditions 1 and 2 of Theorem 10 are fulfilled by $\Lambda$ and $\Theta$. By Theorem 10, $\Lambda$ and $\Theta$ have a unique common fixed point, i.e., $\mu=1$.

The second result of this part is as follows.

Theorem 12. Let $\left(\Pi, \Omega, \Gamma_{\mathfrak{b}}, *\right)$ be a complete FBM-space such that

$$
\lim _{\eta \longrightarrow \infty} \Gamma_{\mathfrak{b}}(\mu, \sigma, \eta)=1 \quad \text { for all } \mu \in \Pi, \sigma \in \Omega
$$

Let $\Lambda: \Pi \cup \Omega \longrightarrow \Pi \cup \Omega$ be two mappings satisfying

(1) $\Lambda(\Pi) \subseteq \Omega, \Lambda(\Omega) \subseteq \Pi$, and $\Theta(\Pi) \subseteq \Omega, \Theta(\Omega) \subseteq \Pi$

(2) $\Gamma_{\mathfrak{b}}(\Lambda(\sigma), \Theta(\mu), k \eta) \geq \Gamma_{\mathfrak{b}}(\mu, \sigma, \eta)$ for all $\mu \in \Pi, \sigma \in \Omega$, and $\eta>0$, where $0<k<1$

Then, $\Lambda$ and $\Theta$ have a unique common fixed point.

Proof. Fix $\mu_{0} \in \Pi$ and $\sigma_{0} \in \Omega$ and assume that $\Lambda\left(\mu_{2 \alpha}\right)=\sigma_{2 \alpha}$, $\Theta\left(\mu_{2 \alpha+1}\right)=\sigma_{2 \alpha+1}, \Lambda\left(\sigma_{2 \alpha}\right)=\mu_{2 \alpha+1}$, and $\Theta\left(\sigma_{2 \alpha+1}\right)=\mu_{2 \alpha+2}$ for all $\alpha \in \mathbb{N} \cup\{0\}$. Then, we get $\left(\mu_{\alpha}, \sigma_{\alpha}\right)$ as a bisequence on the FBM-space $\left(\Pi, \Omega, \Gamma_{\mathfrak{b}}, *\right)$. Now, we have

$$
\Gamma_{\mathfrak{b}}\left(\mu_{1}, \sigma_{0}, \eta\right)=\Gamma_{\mathfrak{b}}\left(\Lambda\left(\sigma_{0}\right), \Theta\left(\mu_{0}\right), \eta\right) \geq \Gamma_{\mathfrak{b}}\left(\mu_{0}, \sigma_{0}, \frac{\eta}{k}\right)
$$

$\forall \eta>0$ and $\alpha \in \mathbb{N}$. By induction, we get

$$
\begin{gathered}
\Gamma_{\mathfrak{b}}\left(\mu_{2 \alpha+1}, \sigma_{2 \alpha+1}, \eta\right)=\Gamma_{\mathfrak{b}}\left(\Lambda\left(\sigma_{2 \alpha}\right), \Theta\left(\mu_{2 \alpha+1}\right), \eta\right) \geq \Gamma_{\mathfrak{b}}\left(\mu_{0}, \sigma_{0}, \frac{\eta}{k^{4 \alpha+1}}\right), \\
\Gamma_{\mathfrak{b}}\left(\mu_{2 \alpha+1}, \sigma_{2 \alpha}, \eta\right)=\Gamma_{\mathfrak{b}}\left(\Lambda\left(\sigma_{2 \alpha}\right), \Theta\left(\mu_{2 \alpha}\right), \eta\right) \geq \Gamma_{\mathfrak{b}}\left(\mu_{0}, \sigma_{0}, \frac{\eta}{k^{4 \alpha}}\right),
\end{gathered}
$$

for all $\eta>0$ and $\alpha \in \mathbb{N}$. Letting $\alpha<\beta$, for $\alpha, \beta \in \mathbb{N}$. Then, from the definition of the fuzzy bipolar metric space, we get

$$
\begin{gathered}
\Gamma_{\mathfrak{b}}\left(\mu_{\alpha}, \sigma_{\beta}, \eta\right) \geq \Gamma_{\mathfrak{b}}\left(\mu_{\alpha}, \sigma_{\alpha}, \frac{\eta}{3}\right) * \Gamma_{\mathfrak{b}}\left(\mu_{\alpha+1}, \sigma_{\alpha}, \frac{\eta}{3}\right) \\
* \Gamma_{\mathfrak{b}}\left(\mu_{\alpha+1}, \sigma_{\beta}, \frac{\eta}{3}\right), \\
\vdots \\
\geq \Gamma_{\mathfrak{b}}\left(\mu_{\alpha}, \sigma_{\alpha}, \frac{\eta}{3}\right) * \Gamma_{\mathfrak{b}}\left(\mu_{\alpha+1}, \sigma_{\alpha}, \frac{\eta}{3}\right) * \cdots * \Gamma_{\mathfrak{b}}\left(\mu_{\beta-1}, \sigma_{\beta-1}, \frac{\eta}{3^{\beta-1}}\right) \\
* \Gamma_{\mathfrak{b}}\left(\mu_{\beta}, \sigma_{\beta-1}, \frac{\eta}{3^{\beta-1}}\right) * \Gamma_{\mathfrak{b}}\left(\mu_{\beta}, \sigma_{\beta}, \frac{\eta}{3^{\beta-1}}\right) .
\end{gathered}
$$

Therefore,

$$
\begin{gathered}
\Gamma_{\mathfrak{b}}\left(\mu_{\alpha}, \sigma_{\beta}, \eta\right) \geq \Gamma_{\mathfrak{b}}\left(\mu_{0}, \sigma_{0}, \frac{\eta}{3 k^{2 \alpha+1}}\right) * \Gamma_{\mathfrak{b}}\left(\mu_{0}, \sigma_{0}, \frac{\eta}{3 k^{2 \alpha}}\right) * \cdots \\
\cdots * \Gamma_{\mathfrak{b}}\left(\mu_{0}, \sigma_{0}, \frac{\eta}{3^{\beta-1} k^{2 \beta+1}}\right) .
\end{gathered}
$$

From (16), as $\alpha, \beta \longrightarrow \infty$, we get

$$
\Gamma_{\mathfrak{b}}\left(\mu_{\alpha}, \sigma_{\beta}, \eta\right) \geq 1 \quad \forall \eta>0 .
$$

Thus, bisequence $\left(\left\{\mu_{\alpha}\right\},\left\{\sigma_{\alpha}\right\}\right)$ is a Cauchy bisequence, since $\left(\Pi, \Omega, \Gamma_{\mathfrak{b}}, \star\right)$ is a complete FBM-space. By Lemma 7 , bisequence $\left(\left\{\mu_{\alpha}\right\},\left\{\sigma_{\alpha}\right\}\right)$ is a biconvergent sequence. Therefore, $\left\{\mu_{\alpha}\right\} \longrightarrow u$ and $\left\{\sigma_{\alpha}\right\} \longrightarrow u$, where $u \in \Pi \cap \Omega$. By Lemma 8 , both sequences $\left\{\mu_{\alpha}\right\}$ and $\left\{\sigma_{\alpha}\right\}$ have a unique limit. From the triangular property of fuzzy bipolar metric spaces, we have

$$
\begin{aligned}
\Gamma_{\mathfrak{b}}(\Lambda(u), u, \eta) \geq & \Gamma_{\mathfrak{b}}\left(\Lambda(u), \sigma_{\alpha+1}, \frac{\eta}{3}\right) * \Gamma_{\mathfrak{b}}\left(\mu_{\alpha+1}, \sigma_{\alpha+1}, \frac{\eta}{3}\right) \\
& * \Gamma_{\mathfrak{b}}\left(\mu_{\alpha+1}, u, \frac{\eta}{3}\right)=\Gamma_{\mathfrak{b}}\left(\Lambda(u), \Theta\left(\mu_{\alpha+1}\right), \frac{\eta}{3}\right) \\
& * \Gamma_{\mathfrak{b}}\left(\Lambda \sigma_{\alpha}, \Theta \mu_{\alpha+1}, \frac{\eta}{3}\right) * \Gamma_{\mathfrak{b}}\left(\mu_{\alpha+1}, u, \frac{\eta}{3}\right) \\
\geq & \Gamma_{\mathfrak{b}}\left(u, \mu_{\alpha+1}, \frac{\eta}{3 k}\right) * \Gamma_{\mathfrak{b}}\left(\sigma_{\alpha}, \mu_{\alpha+1}, \frac{\eta}{3 k}\right) \\
& * \Gamma_{\mathfrak{b}}\left(\mu_{\alpha+1}, u, \frac{\eta}{3}\right),
\end{aligned}
$$

for all $\alpha \in \mathbb{N}$ and $\eta>0$ and as $\alpha \longrightarrow \infty$,

$$
\Gamma_{\mathfrak{b}}(\Lambda(u), u, \eta) \longrightarrow 1 * 1 * 1=1 .
$$

From Definition 3 condition (2), $\Lambda(u)=u$. Again,

$$
\Gamma_{\mathfrak{b}}(u, \Theta(u), \eta)=\Gamma_{\mathfrak{b}}(\Lambda(u), \Theta(u), \eta) \geq \Gamma_{\mathfrak{b}}\left(u, u, \frac{\eta}{k}\right)=1
$$

Therefore, $\Theta(u)=u$. Hence, $u$ is common fixed point of $\Lambda$ and $\Theta$. Let $v \in \Pi \cap \Omega$ be a another fixed point of $\Lambda$ and $\Theta$. Then,

$$
\Gamma_{\mathfrak{b}}(u, v, \eta)=\Gamma_{\mathfrak{b}}(\Lambda(u), \Theta(v), \eta) \geq \Gamma_{\mathfrak{b}}\left(u, v, \frac{\eta}{k}\right)
$$

for $0<k<1$ and $\forall \eta>0$. By Lemma 5 , we have $u=v$.

To support the above theorem, we present the following example.

Example 13. Let $\Pi=\{0,1,2,7\}$ and $\Omega=\{0,1 / 3,1 / 2,3\}$ and define a continuous $\eta$-norm as $r * \zeta=\min \{r, \zeta\}$. Define $\Gamma_{\mathfrak{b}}(\mu, \sigma, \eta)=\exp ^{-}(|\mu-\sigma| / \eta)$ for all $\eta>0, \mu \in \Pi$, and $\sigma \in \Omega$. Then, $\left(\Pi, \Omega, \Gamma_{\mathfrak{b}}, *\right)$ is a complete FBM-space. Suppose we 
define a mapping $\Lambda, \Theta: \Pi \cup \Omega \longrightarrow \Pi \cup \Omega$ by

$$
\begin{aligned}
& \Lambda(\mu)= \begin{cases}\frac{1}{3}, & \text { if } \mu \in\{7,2\}, \\
0, & \text { if } \mu \in\left\{0, \frac{1}{3}, \frac{1}{2}, 1,3\right\},\end{cases} \\
& \Theta(\mu)= \begin{cases}\frac{1}{2}, & \text { if } \mu \in\{7,2\}, \\
0, & \text { if } \mu \in\left\{0, \frac{1}{3}, \frac{1}{2}, 1,3\right\} .\end{cases}
\end{aligned}
$$

Now, suppose that $k=1 / 2$, then for all $\eta>0$, we obtain the following cases.

Case 1. Let $\mu \in\{7,2\}$ and $\sigma \in\{0,1 / 3,1 / 2,1,3\}$, then

$$
\begin{aligned}
\Gamma_{\mathfrak{b}}(\Lambda(\mu), \Theta(\sigma), k \eta) & =\Gamma_{\mathfrak{b}}\left(\frac{1}{3}, 0, k \eta\right)=\frac{k \eta}{k \eta+|1 / 3|} \\
& \geq \frac{\eta}{\eta+|\mu-\sigma|}=\Gamma_{\mathfrak{b}}(\mu, \sigma, \eta) .
\end{aligned}
$$

Case 2. Let $\mu \in\{0,1 / 3,1 / 2,1,3\}$ and $\sigma \in\{7,2\}$, then

$$
\begin{aligned}
\Gamma_{\mathfrak{b}}(\Lambda(\mu), \Theta(\sigma), k \eta) & =\Gamma_{\mathfrak{b}}\left(0, \frac{1}{2}, k \eta\right)=\frac{k \eta}{k \eta+|1 / 2|} \\
& \geq \frac{\eta}{\eta+|\mu-\sigma|}=\Gamma_{\mathfrak{b}}(\mu, \sigma, \eta) .
\end{aligned}
$$

Therefore, the conditions 1 and 2 of Theorem 12 were also satisfied by $\Lambda$ and $\Theta$. Based on Theorem 12, we get $\Lambda$ and $\Theta$ that have a unique common fixed point, i.e., $\mu=0$.

\section{Supportive Application}

In this section, we apply Theorem 10 to discuss the existence and uniqueness solution to the following nonlinear integral equations:

$$
\left\{\begin{array}{l}
\mu(\gamma)=\mathfrak{b}(\gamma)+\int_{\mathscr{C}_{1} \cup \mathscr{C}_{2}} \mathscr{G}_{1}(\gamma, \zeta, \mu(\zeta)) d \zeta, \gamma \in \mathscr{E}_{1} \cup \mathscr{E}_{2}, \\
\mu(\gamma)=\mathfrak{b}(\gamma)+\int_{\mathscr{C}_{1} \cup \mathscr{C}_{2}} \mathscr{G}_{2}(\gamma, \zeta, \mu(\zeta)) d \zeta, \gamma \in \mathscr{E}_{1} \cup \mathscr{E}_{2},
\end{array}\right.
$$

where $\mathscr{E}_{1} \cup \mathscr{E}_{2}$ is a Lebesgue measurable set with $m\left(\mathscr{E}_{1} \cup\right.$ $\left.\mathscr{E}_{2}\right)<\infty$. Let $\Pi=L^{\infty}\left(\mathscr{E}_{1}\right)$ and $\Omega=L^{\infty}\left(\mathscr{E}_{2}\right)$ be two normed linear spaces. Define $\left.\Gamma_{\mathfrak{b}}: \Pi \times \Omega \times(0, \infty) \longrightarrow 0,1\right]$ by

$$
\left.\Gamma_{\mathfrak{b}}(\mu, \sigma, \eta)=e^{-\left(\sup _{\gamma \in \mathscr{E}_{1} \cup \mathscr{E}_{2}}|\mu(\gamma)-\sigma(\gamma)| / \eta\right.}\right),
$$

for all $\mu \in \Pi, \sigma \in \Omega$. Clearly, $\left(\Pi, \Omega, \Gamma_{\mathfrak{b}}, *\right)$ is a complete FBMspace.
System (29) will be considered under the following hypotheses:

(i) $\left.\left.\mathscr{G}_{1}, \mathscr{G}_{2}:\left(\mathscr{E}_{1}^{2} \cup \mathscr{E}_{2}^{2}\right) \times 0, \infty\right) \longrightarrow 0, \infty\right)$ and $b \in L^{\infty}($ $\left.\mathscr{E}_{1}\right) \cup L^{\infty}\left(\mathscr{E}_{2}\right)$

(ii) There is a continuous function $\theta: \mathscr{E}_{1}^{2} \cup \mathscr{E}_{2}^{2} \longrightarrow 0$, $\infty$ and $k \in(0,1)$ such that $\mid \mathscr{G}_{1}(\gamma, \zeta, \mu(\zeta))-\mathscr{G}_{2}(\gamma, \zeta$ , $\sigma(\zeta)) \mid \leq k \theta(\gamma, \zeta)(|\mu(\gamma)-\sigma(\gamma)|)$, for $\gamma, \zeta \in \mathscr{E}_{1}^{2} \cup \mathscr{E}_{2}^{2}$

(iii) $\sup _{\gamma \in \mathscr{E}_{1} \cup \mathscr{E}_{2}} \int_{\mathscr{E}_{1} \cup \mathscr{E}_{2}} \theta(\gamma, \zeta) d \zeta \leq 1$

Theorem 14. Under hypotheses (i)-(iii), System (29) has a unique common solution in $L^{\infty}\left(\mathscr{E}_{1}\right) \cup L^{\infty}\left(\mathscr{E}_{2}\right)$.

Proof. Define the mappings $\Lambda, \Theta: L^{\infty}\left(\mathscr{E}_{1}\right) \cup L^{\infty}\left(\mathscr{E}_{2}\right) \longrightarrow$ $L^{\infty}\left(\mathscr{E}_{1}\right) \cup L^{\infty}\left(\mathscr{E}_{2}\right)$ by

$$
\begin{aligned}
& \Lambda(\mu(\gamma))=\mathfrak{b}(\gamma)+\int_{\mathscr{E}_{1} \cup \mathscr{C}_{2}} \mathscr{G}_{1}(\gamma, \zeta, \mu(\zeta)) d \zeta, \gamma \in \mathscr{E}_{1} \cup \mathscr{E}_{2}, \\
& \Theta(\mu(\gamma))=\mathfrak{b}(\gamma)+\int_{\mathscr{E}_{1} \cup \mathscr{C}_{2}} \mathscr{G}_{2}(\gamma, \zeta, \mu(\zeta)) d \zeta, \gamma \in \mathscr{E}_{1} \cup \mathscr{E}_{2} .
\end{aligned}
$$

Now, we have

$$
\begin{aligned}
& \Gamma_{\mathfrak{b}}(\Lambda \mu(\gamma), \Theta \sigma(\gamma), k \eta) \\
& -\sup _{\gamma \in \mathscr{E}_{1} \cup \mathscr{E}_{2}}(|\Lambda \mu(\gamma)-\Theta \sigma(\gamma)| / k \eta) \\
& =e^{\gamma \in \mathscr{E}_{1} \cup \mathscr{E}} \\
& =e^{\left.-\sup _{\gamma \in \mathscr{E}_{1} \cup \mathscr{C}_{2}}\left(\mid \mathfrak{b}(\gamma)+\int_{\mathscr{E}_{1} \cup \mathscr{E}_{2}} \mathscr{G}_{1}(\gamma, \zeta, \mu(\zeta)) d \zeta-\mathfrak{b}(\gamma)-\int_{\mathscr{E}_{1} \cup \mathscr{E}_{2}} \mathscr{G}_{2}(\gamma, \zeta, \sigma(\zeta)) d \zeta\right) \mid / k \eta\right)} \\
& =e^{-\sup _{\gamma \in \mathscr{E}_{1} \cup \mathscr{G}_{2}}\left(\left|\mathfrak{b}(\gamma)+\int_{\mathscr{E}_{1} \cup \mathscr{E}_{2}} \mathscr{G}_{1}(\gamma, \zeta, \mu(\zeta)) d \zeta-\left(\mathfrak{b}(\gamma)+\int_{\mathscr{E}_{1} \cup \mathscr{E}_{2}} \mathscr{G}_{2}(\gamma, \zeta, \sigma(\zeta)) d \zeta\right)\right| / k \eta\right)} \\
& \geq e^{-\sup _{\gamma \in \mathscr{E}_{1} \cup \mathscr{E}_{2}}\left(\int_{\mathscr{E}_{1} \cup \mathscr{E}_{2}}\left|\mathscr{G}_{1}(\gamma, \zeta, \mu(\zeta))-\mathscr{G}_{2}(\gamma, \zeta, \sigma(\zeta))\right| d \zeta / k \eta\right)} \\
& \left.\geq e^{-\sup _{\gamma \in \mathscr{E}_{1} \cup \mathscr{E}_{2}}\left(\int_{\mathscr{E}_{1} \cup \mathscr{G}_{2}} k \theta(\gamma, \zeta)(|\mu(\gamma)-\sigma(\gamma)|) d \zeta / k \eta\right.}\right) \\
& \geq e^{-\sup _{\gamma \in \mathscr{E}_{1} \cup \mathscr{E}_{2}}\left(\int_{\mathscr{E}_{1} \cup \mathscr{E}_{2}} k \theta(\gamma, \zeta)(|\mu(\gamma)-\sigma(\gamma)|) d \zeta / k \eta\right)} \\
& \geq e^{-\sup _{\gamma \in \mathscr{E}_{1} \cup \mathscr{E}_{2}}(|\mu(\gamma)-\sigma(\gamma)| / \eta)}=\Gamma_{\mathfrak{b}}(\mu, \sigma, \eta) \text {. }
\end{aligned}
$$

Hence, all hypotheses of Theorem 10 are fulfilled, and consequently, the system (29) has a unique common solution.

Example 15. Let $\mathscr{E}_{1}=[0,1], \mathscr{E}_{2}=[1,2], \Pi=L^{\infty}\left(\mathscr{E}_{1}\right)$, and $\Omega$ $=L^{\infty}\left(\mathscr{E}_{2}\right)$. Now, consider the following nonlinear integral equations as 


$$
\begin{aligned}
& \Lambda(\mu(\gamma))=\gamma+\int_{\mathscr{E}_{1} \cup \mathscr{E}_{2}}\left(\gamma+\zeta+\frac{\mu(\zeta)}{8(1+\mu(\zeta))}\right) d \zeta \\
& \Theta(\mu(\gamma))=\gamma+\int_{\mathscr{E}_{1} \cup \mathscr{E}_{2}}\left(\gamma+\zeta+\frac{\mu(\zeta)}{8(1+\mu(\zeta))}\right) d \zeta
\end{aligned}
$$

for all $\gamma \in \mathscr{E}_{1} \cup \mathscr{E}_{2}$. Then clearly, the above equation is in the form of the following equation:

$$
\begin{array}{ll}
\Lambda(\mu(\gamma))=\mathfrak{b}(\gamma)+\int_{\mathscr{E}_{1} \cup \mathscr{C}_{2}} \mathscr{G}_{1}(\gamma, \zeta, \mu(\zeta)) d \zeta, & \gamma \in \mathscr{E}_{1} \cup \mathscr{E}_{2}, \\
\Theta(\mu(\gamma))=\mathfrak{b}(\gamma)+\int_{\mathscr{E}_{1} \cup \mathscr{C}_{2}} \mathscr{G}_{2}(\gamma, \zeta, \mu(\zeta)) d \zeta, & \gamma \in \mathscr{E}_{1} \cup \mathscr{E}_{2},
\end{array}
$$

where $\mathfrak{b}(\gamma)=\gamma$ and

$$
\begin{aligned}
& \mathscr{G}_{1}(\gamma, \zeta, \mu(\zeta))=\gamma+\zeta+\frac{\mu(\zeta)}{8(1+\mu(\zeta))}, \\
& \mathscr{G}_{2}(\gamma, \zeta, \mu(\zeta))=\gamma+\zeta+\frac{\mu(\zeta)}{8(1+\mu(\zeta))}
\end{aligned}
$$
have

That is, (33) is a particular case of system (29). Now, we

$$
\begin{aligned}
& \left|\mathscr{G}_{1}(\gamma, \zeta, \mu(\zeta))-\mathscr{G}_{2}(\gamma, \zeta, \sigma(\zeta))\right| \\
& =\left|\frac{\mu(\zeta)}{8(1+\mu(\zeta))}-\frac{\sigma(\zeta)}{8(1+\sigma(\zeta))}\right| \\
& =\left|\frac{1}{8} \frac{\mu(\zeta)-\sigma(\zeta)}{(1+\mu(\zeta))(1+\sigma(\zeta))}\right| \\
& \leq \frac{1}{8}(|\mu(\zeta)-\sigma(\zeta)|) .
\end{aligned}
$$

Consider a continuous function $\theta: \mathscr{E}_{1}^{2} \cup \mathscr{E}_{2}^{2} \longrightarrow[0, \infty)$ defined by $\theta(\gamma, \zeta)=1 / 4 \forall \gamma, \zeta \in \mathscr{E}_{1}^{2} \cup \mathscr{E}_{2}^{2}$. Then, we obtain

$$
\sup _{\gamma \in \mathscr{E}_{1} \cup \mathscr{E}_{2}} \int_{\mathscr{E}_{1} \cup \mathscr{E}_{2}} \theta(\gamma, \zeta) d \zeta \leq 1
$$

Therefore, all the conditions of Theorem 14 are satisfied. Hence, system (33) has a unique common solution in $L^{\infty}\left(\mathscr{E}_{1}\right) \cup L^{\infty}\left(\mathscr{E}_{2}\right)$.

\section{Conclusion}

First of all, we proved common fixed point theorems on fuzzy bipolar metric space with an application. On the basis of the ideas of this paper along with the literature present on FBM-spaces, we encourage the interested researcher to explore more interesting results for these spaces.

\section{Data Availability}

No data were used to support this study.

\section{Conflicts of Interest}

The authors declare that there are no conflicts of interest regarding the publication of this paper.

\section{Authors' Contributions}

All authors contributed equally in this research article. All authors read and approved the final manuscript.

\section{References}

[1] B. Schweizer and A. Sklar, "Statistical metric spaces," Pacific Journal of Mathematics, vol. 10, no. 1, pp. 313-334, 1960.

[2] L. A. Zadeh, "Fuzzy sets," Information and Control, vol. 8, no. 3, pp. 338-353, 1965.

[3] V. Ivanova and B. Zlatanov, "Implementation of fuzzy functions aimed at fairer grading of students' tests," Education Sciences, vol. 9, no. 3, p. 214, 2019.

[4] V. Ivanova and B. Zlatanov, "Application of fuzzy logic in online test evaluation in English as a foreign language at university level," AIP Conference Proceedings, vol. 2172, no. 1, 2019.

[5] V. Gregori and A. Sapena, "On fixed-point theorems in fuzzy metric spaces," Fuzzy Sets and Systems, vol. 125, no. 2, pp. 245-252, 2002.

[6] N. Rusmiari, D. Putra, and A. Sasmita, "Fuzzy logic method for evaluation of difficulty level of exam and student graduation," International Journal of Computer Science, vol. 10, no. 2, pp. 223-229, 2013.

[7] I. Kramosil and J. Michalek, "Fuzzy metric and statistical metric spaces," Kybernetica, vol. 11, pp. 326-334, 1975.

[8] A. George and P. Veeramani, "On some results in fuzzy metric spaces,” Fuzzy Sets and Systems, vol. 64, no. 3, pp. 395-399, 1994.

[9] M. Grabiec, "Fixed points in fuzzy metric spaces," Fuzzy Sets and Systems, vol. 27, no. 3, pp. 385-389, 1988.

[10] V. Gregori and A. Sapena, "On fixed-point theorems in fuzzy metric spaces," Fuzzy Sets and Systems, vol. 125, no. 2, pp. 245-252, 2002.

[11] X. Li, S. U. Rehman, S. U. Khan, H. Aydi, N. Hussain, and J. Ahmad, "Strong coupled fixed point analysis in fuzzy metric spaces with application to Urysohn integral equations," Dynamic Systems and Applications, vol. 30, no. 2, pp. 197218, 2002.

[12] S. Beloul and A. Tomar, "Integral type common fixed point theorems in modified intuitionistic fuzzy metric spaces," Afrika Matematika, vol. 30, no. 3-4, pp. 581-596, 2019.

[13] G. Prasad, A. Tomar, R. C. Dimri, and A. Bartwal, "Coincidence theorems via contractive mappings in ordered nonArchimedean fuzzy metric spaces," Journal of the Korean Society of Mathematical Education Series B: The Pure and Applied Mathematiccs, vol. 27, no. 4, pp. 187-205, 2020.

[14] G. Prasad, "Coincidence points of relational $\psi$-contractions and an application," Afrika Matematika, vol. 32, no. 7-8, pp. 1475-1490, 2021.

[15] A. Mutlu, K. Özkan, and U. Gürdal, "Fixed point theorems for multivalued mappings on bipolar metric spaces," Fixed Point Theory, vol. 21, no. 1, pp. 271-280, 2020. 
[16] Y. U. Gaba, M. Aphane, and V. Sihag, "On two Banach-type fixed points in bipolar metric spaces," Abstract and Applied Analysis, vol. 2021, Article ID 4846877, 10 pages, 2021.

[17] Y. U. Gaba, M. Aphane, and H. Aydi, " $(\alpha, \mathrm{BK})$-Contractions in bipolar metric spaces," Journal of Mathematics, vol. 2021, Article ID 5562651, 6 pages, 2021.

[18] G. N. V. Kishore, K. P. R. Rao, A. Sombabu, and R. V. N. S. Rao, "Related results to hybrid pair of mappings and applications in bipolar metric spaces," Journal of Mathematics, vol. 2019, Article ID 8485412, 7 pages, 2019.

[19] A. Mutlu and U. Gurdal, "Bipolar metric spaces and some fixed point theorems," Journal of Nonlinear Sciences and Applications, vol. 9, no. 9, pp. 5362-5373, 2016.

[20] A. Bartwal, R. C. Dimri, and G. Prasad, "Some fixed point theorems in fuzzy bipolar metric spaces," Journal of Nonlinear Sciences and Applications, vol. 13, no. 4, pp. 196-204, 2020.

[21] S. N. Mishra, N. Sharma, and S. L. Singh, "Common fixed points of maps on fuzzy metric spaces," International Journal of Mathematics and Mathematical Sciences, vol. 17, no. 2, 258 pages, 1994. 